\title{
Advances in Theories and Methods of Smoking Cessation Services in Community
}

\author{
Qiao Xiong \\ Preventive Medicine, Capital Medical University, Beijing, China \\ Email: xiongqiao2019hk@163.com
}

How to cite this paper: Xiong, Q. (2019) Advances in Theories and Methods of Smoking Cessation Services in Community. Open Access Library Journal, 6: e5420. https://doi.org/10.4236/oalib.1105420

Received: April 23, 2019

Accepted: May 13, 2019

Published: May 16, 2019

Copyright $\odot 2019$ by author(s) and Open Access Library Inc.

This work is licensed under the Creative Commons Attribution International License (CC BY 4.0).

http://creativecommons.org/licenses/by/4.0/

\begin{abstract}
With the continuous development of the national economy, people's living standards have been constantly improved. According to incomplete statistics, the proportion of smokers is increasing year by year and the number is increasing. Although tobacco sales account for a large proportion of China's tax revenue, the diseases caused by smoking pose a serious challenge to people's health every year. Meanwhile, the medical expenses incurred are also increasing. At present, China's society has begun to gradually transform into an ecological type. Therefore, it is urgent to comprehensively increase tobacco control measures. In the life structure of Chinese residents, community is an important link. Making full use of community resources to carry out community smoking cessation services has become an important and convenient task.
\end{abstract}

\author{
Subject Areas \\ Public Health \\ Keywords \\ Community, Smoking Cessation, Smoking Control, Theory, Method
}

\section{Overall Investigation}

According to the comprehensive survey of the World Health Organization, China has become the largest tobacco consumer in the world. At the same time, China is one of the few countries in the world where the smoking rate continues to rise. According to the production and sales data of China National Tobacco Corporation, the cigarettes produced by the company accounted for more than $90 \%$ of domestic consumption, and also accounted for nearly half of global consumption [1]. From the perspective of the harmfulness of smoking, the number of deaths caused by smoking worldwide exceeds 4 million per year, while in 
China, according to incomplete statistics, this number is also as high as more than 1 million [2]. It can be seen that smoking poses a serious challenge to the healthy life of residents, and how to effectively carry out smoking cessation work has become an important research topic. In this study, we mainly focus on how to carry out smoking cessation services in community.

\section{Basic Concept}

Tobacco damage to the human body mainly includes nicotine, tar and carbon monoxide, and the human body's addiction to tobacco refers to a substance dependence on the harmful components in tobacco, mainly nicotine. It can be seen that the main task and purpose of smoking cessation is to cut off this dependence, which should be cut off not only formally but also spiritually [3], However, according to the current social research, "mental smoking cessation" is more difficult.

In China's residential system, community is a very important part, and it is easier and more popular to carry out smoking cessation services in the community. Community health education takes community residents as the main target, and takes improving of residents' physical health as the basic goal. The ultimate purpose of community smoking cessation services and help is to promote community residents to establish health awareness, develop healthy behaviors and lifestyles, enable residents to actively participate in community tobacco control activities, get familiar with and master smoking cessation methods and skills, and discourage friends or family members from smoking, so as to improve the overall health level of the community [4].

\section{Basic Ideas for Smoking Cessation Services}

Through the analysis of the causes of smoking addiction and the research on the composition of the community in our country, we should set up a special organization, improve the corresponding management system, publicity and training programs, regular investigation and follow-up system, and on this basis, to explore and study the basic method of smoking cessation in the community smoking cessation service. It has been shown that the basic idea of quitting smoking is to break the addiction to nicotine. However, through investigation, the current methods of quitting smoking generally include intervention in smoking cessation, counseling to quit smoking, drug quitting, etc. [5], while working in the community, Considering all kinds of influencing factors comprehensively, we can use methods such as counseling to quit smoking and intervention in smoking cessation. If conditions permit, we can quit smoking by drugs.

\section{The Implementation and Basic Progress of Community Smoking Cessation Services}

\subsection{Improve Service Plans and Strengthen Community Management}

In order to carry out the community smoking cessation service work in an or- 
derly, high-quality and efficient way, it is necessary to develop a relatively perfect service scheme or management system. First of all, Community leadership should pay sufficient attention to the work and establish a corresponding management organization or network. The person in charge of the community should be the member of the community leadership team, and they should set up part-time tobacco control staff in each neighborhood committee to cooperate with the street to conduct investigate and survey, propaganda, information communication, data statistics, data distribution, etc.; secondly, we must improve the rules and regulations, and establish and improve the tobacco control rules and regulations with the tobacco control organization as the core, starting from caring for the health of community residents, to carry out smoke-free areas and smoking cessation publicity day in a way of implementing the concept of smoking cessation into the minds of residents [6].

\subsection{Follow-Up Investigation at Regular Intervals and Strengthen Publicity and Training}

On the basis of improving the organization and rules and regulations of community smoking cessation services, it is important to carry out two tasks: follow-up investigation and publicity and training. For the follow-up survey, the purpose is to timely grasp the health status of residents in the community, the understanding of residents in the community about tobacco knowledge and tobacco control, the attitude and initiative of residents towards tobacco control, as well as the demand and access to smoking cessation services [7]. The smoking cessation organization should regularly arrange staff members to grasp relevant basic dynamics and data in a timely manner through questionnaires, face-to-face interviews, telephone follow-ups and WeChat communication, and grasp the key points according to the information and data of the survey statistics. In doing so, scientific decision-making can be made, which lay a good foundation for the next step. Secondly, we must do a good job in publicity and training. At present, the types of information exchange platforms are very rich. On the basis of the past banners, blackboard newspapers and publicity boards, we can also make full use of community public TV and community WeChat platform to open up new propaganda columns, attracting as many residents as possible into the smoking cessation campaign with novel content and form.

\subsection{Several Smoking Cessation Methods}

There are many ways to quit smoking. In this paper, we mainly focus on the specific situation of community service and discuss several ways of smoking cessation such as counseling, intervention and drug [8].

Counseling smoking cessation; In combination with the actual situation of the community, the most cost-effective way is to take counseling to quit smoking, which generally includes telephone counseling, individual behavior counseling and professional counseling. Through telephone counseling, it is easy to provide smoking cessation knowledge and help intervention for quitters, and get timely 
feedback on smoking cessation progress. In actual application, a survey in a city indicates that the efficiency of smoking cessation has been improved to some extent through telephone counseling. As the number of calls increased, so does the success rate; For communities with conditions, individual behavior counseling or professional counseling can be carried out, which can improve the efficiency of quitting smoking to a certain extent [9].

Intervention smoking cessation; in recent years, with the continuous progress of science and technology, various means of smoking cessation have emerged in an endless stream. However, in community service, in addition to consulting smoking cessation, a more economical and practical method is to intervene to quit smoking. Foreign foreigners such as Lawson PJ summarized the intervention to quit smoking as " $5 \mathrm{~A}$ " method: Ask Advice, Assess, Assist and Arrange. The 5A method can be used to intervene smokers systematically. Especially for those who are not willing to give up smoking subjectively, it is an effective and reasonable way to persuade them to give up smoking as much as possible and improve their subjective initiative to quit smoking through 5A method if their subjective initiative is poor or even with drugs.

Drugs smoking cessation; Compared with the above two methods of smoking cessation, the method is simply to rely on drugs to block the dependence on harmful substances such as nicotine. The continuous application of this method also shows that it is still quite difficult to quit smoking relying only on subjective initiative and external intervention, therefore, if conditions permit-human health conditions and community economic conditions-can choose to smoking cessation with drugs, but the smoking cessation method must be carried out under the guidance and supervision of community medical professionals [10]. In general, drugs smoking cessation are generally used to replace nicotine, reducing the tension and anxiety caused by smoking cessation, so as to achieve the goal of smoking cessation. However, this method also has a big defect. At present, there is still a lack of effective and inexpensive drugs on the market. Therefore, this method is largely restricted by economic conditions [11].

\subsection{Expected Effect Analysis}

With the hard work and efforts of the smoking cessation organization, through the publicity and education and regular intervention, the subjective initiative of quitters in the smoking population must be improved. The extent of the improvement is directly related to the development of community work. First, through the investigation and analysis of previous public data on smoking cessation, as long as community work is carried out seriously and actively, the success rate of smoking cessation will generally increase by more than 8 percentage points [12]; second, the success rate of smoking cessation of male residents rises. After the analysis of public data, generally speaking, male groups with higher education and better career have higher success rate of quitting smoking [13]; Third, no matter whether the success rate of smoking cessation increases or not, 
the implementation of smoking cessation work undoubtedly increases the community residents' awareness of the harm of smoking, and improves the prevalence of smoking cessation knowledge [14].

\section{Conclusions and Guidance Applicable to Chinese Communities}

Through the implementation of community smoking cessation services, we not only make full use of various resources under the jurisdiction of the community, responding to the relevant tobacco control policies, but also greatly mobilize the enthusiasm of residents in the community to quit smoking, creating a more smoke-free environment. However, through this analysis and research, we should strengthen several tasks in our country: first, we must strengthen youth education. Whether parents smoke or not is off the table, but we must guide parents or direct young people to educate young people not to smoke and raise awareness of the harmful effects of smoking [15]; second, we must actively strive for policy support. When conditions permit, it is necessary to actively communicate and coordinate with government departments, and strive for as many tobacco control areas or policies as possible, so as to urge the implementation of smoking cessation work as a strict standard.

\section{Conflicts of Interest}

The author declares no conflicts of interest regarding the publication of this paper.

\section{References}

[1] Editor of the Road to Health (2005) Smoking Always Caused Cancer Quietly, the Road to Health.

[2] Yan, Y., Dong, B. and Yin, X. (2008) Evidence-Based Medicine Evidence for Smoking Cessation Methods. Modern Preventive Medicine, 35, 3118-3121.

[3] Sun, L. and Wei, L. (2011) Thoughts on the Current Smoking Cessation Problem. Medical Sociology, No. 10, 36-46.

[4] Xing, H. (2007) Multiple Correspondence Analysis of Failure Reasons to Smoking Cessation for Rural Residents in Zhejiang Province. Chinese Rural Health Service Administration, 27, 503-505.

[5] Zheng, Y. and Zhao, Y. (2010) Attempt to Analyze the Methods and Problems of Smoking Cessation in China. China Electric Power Education, No. s1, 260-261.

[6] Xu, Z. (2006) Exploration of Health Education Model of Community Smoking Prevention. Chinese Journal of Health Education, 22, 314-316.

[7] Yang, J., Chen, Y., Gao, F., Chang, G., Shan, X. and Zou, X. (2014) Survey and Analysis on Smoking Cessation Counselor's Knowledge for Tobacco Control. China Cancer, No. 6, 473-477.

[8] Wu, L., Jiang, B., He, Y. and Zuo, F. (2014) Progress Research on Smoking Cessation Intervention Models and Methodsat Home and Abroad. Chinese Journal of Health Care and Medicine, 16.

[9] Zeng, H., Fu, S., Liu, Y., Zhu, S. and Jiang, S. (2014) Smoking Cessation Interven- 
tions and Their Application in Primary Care. Chinese General Practice, 17, 1235-1237.

[10] Fu, Y. and Wang, S. (2014) Application of Improving Community Education Mode Based on Smoking Related Theory. Journal of Practical Cardiovascular and Pulmonary Vascular Diseases, 4, 99.

[11] Li, X. (2014) Preliminary Study on Smoking Cessation Methods with Different Educational Levels. Journal of Aerospace Medicine, No. 6, 760-762.

[12] Zhang, H., Shen, C., Zhang, Z., Song, G. and Zhang, Z. (2006) Tianjin Participants' Smoking Cessation Effect Evaluation in International Smoking Cessation Competition. China Chronic Disease Prevention and Control, 3, 179-181.

[13] Li, L. and Xia, Y. (2012) Investigation and Analysis on the Eating Habits and Health of People in Changsha. China Food and Nutrition, 18.

[14] Li, C. (2006) Investigation and Discussion on Health Education Intervention Measures on Smoking of Middle School Students in Haikou City. China Tropical Medicine, 6, 1713-1714.

[15] Zhang, Y., Huang, Y. and Wang, X. (2008) Investigation and Analysis on Smoking Status of Middle School Students. Chinese Journal of Public Health Management, 24, 194-195. 